\title{
Transmission Expansion Planning
}

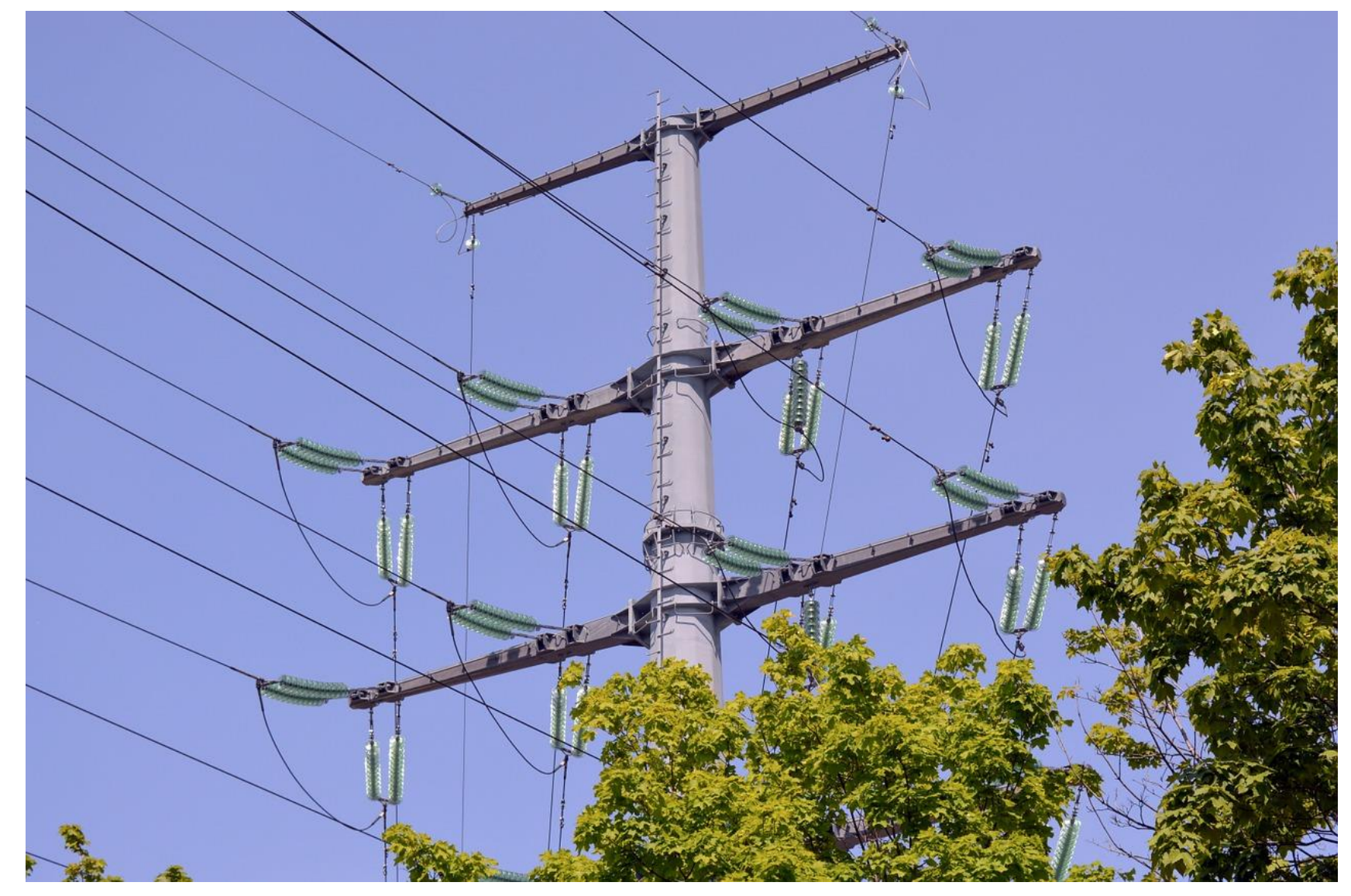

A. J. Conejo, 2018

The OHio State UnIVersity 


\section{What}

1. Transmission Expansion Planning (TEP): Basics

2. TEP via stochastic programming

3. TEP via robust optimization 
Static or single-stage model

Beginning of planning horizon<smiles>[CH2][CH2]</smiles>

\section{Transmission} Expansion Planning

\section{Dynamic or multi-stage model}

Beginning of

planning horizon
End of planning horizon

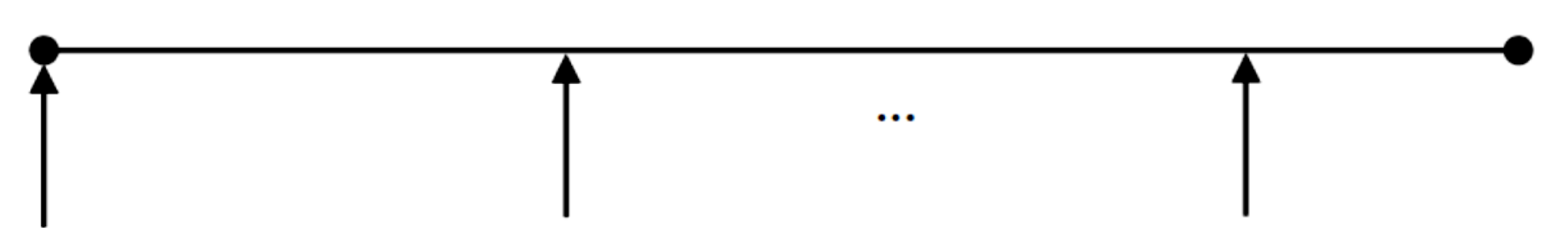
1st transmission
2nd transmission expansion decisions
expansion decisions
nth transmission expansion decisions

End of planning horizon 


\section{Transmission Expansion Planning - Example}

One generating unit at node 1 . Its production cost is $C$, and its capacity infinite.

One demand at node 2 with a peak load $P^{\mathrm{D}^{\max }}$ and infinite load-shedding cost.

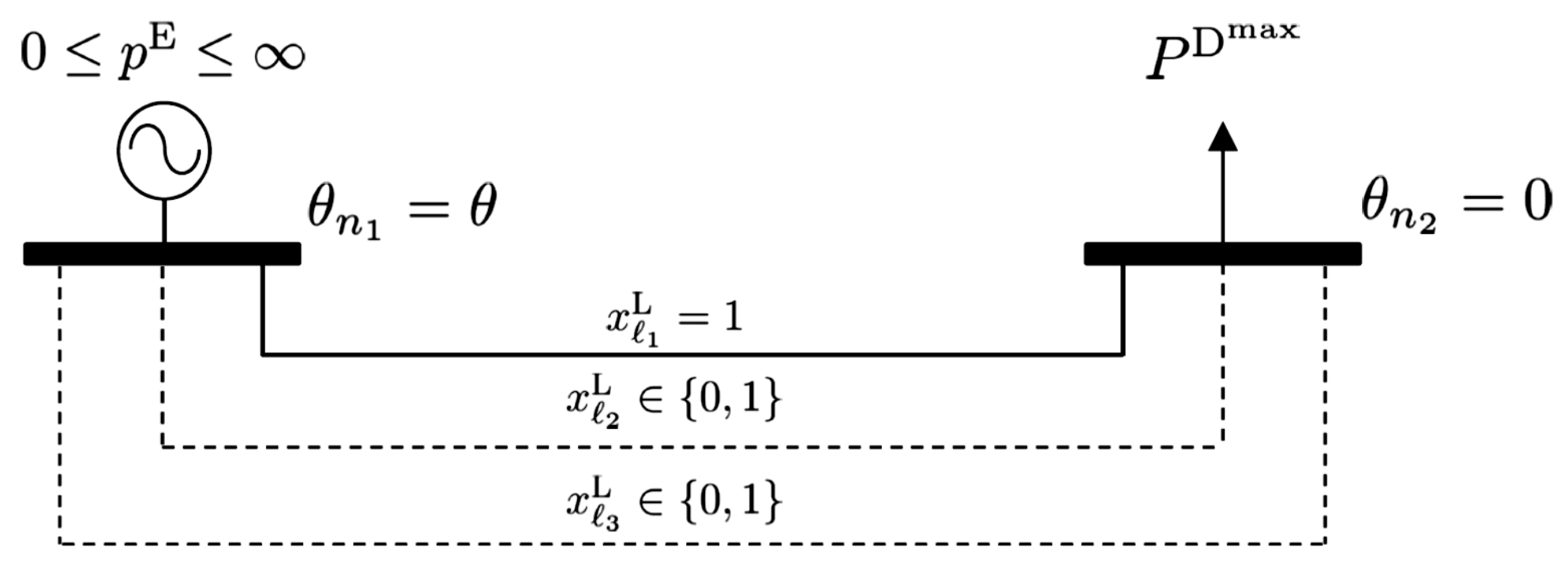




\section{Transmission Expansion Planning}

$0 \leq p^{\mathrm{E}} \leq \infty$ $P^{\mathrm{D} \max }$

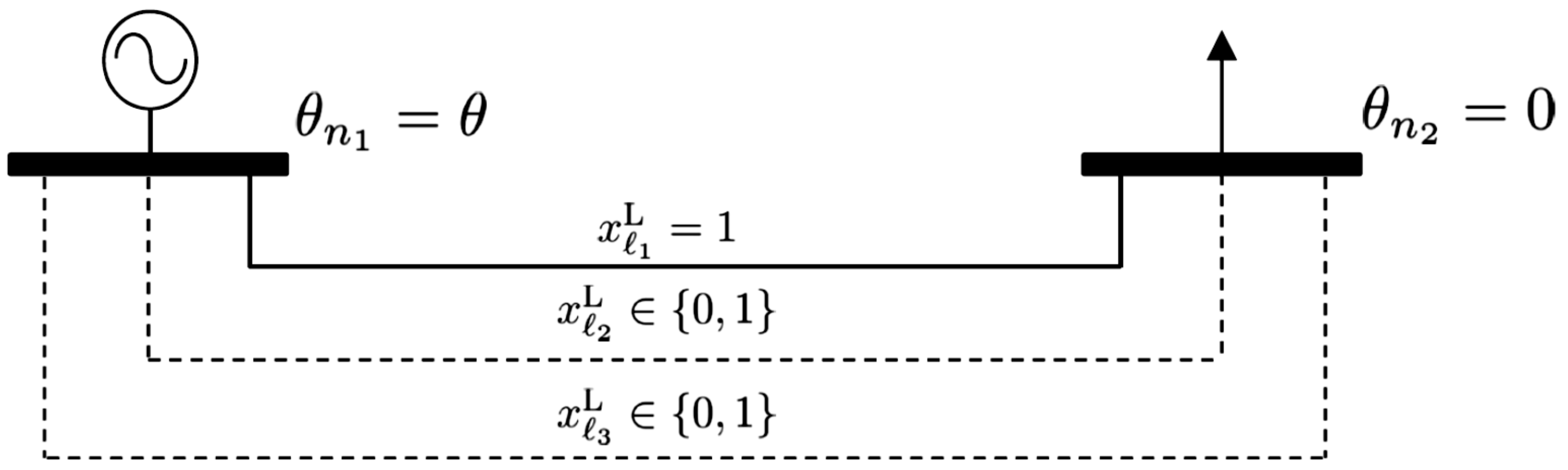




\section{Transmission Expansion Planning}

Nodes 1 and 2 are connected through a transmission line $\ell_{1}$ of capacity $F^{\max }$ and susceptance 1 p.u.

It is possible to build two additional lines $\left(\ell_{2}\right.$ and $\left.\ell_{3}\right)$ equal to the existing one with an annualized investment cost of $I$.

The budget is unlimited.

Node 2 is the reference node.

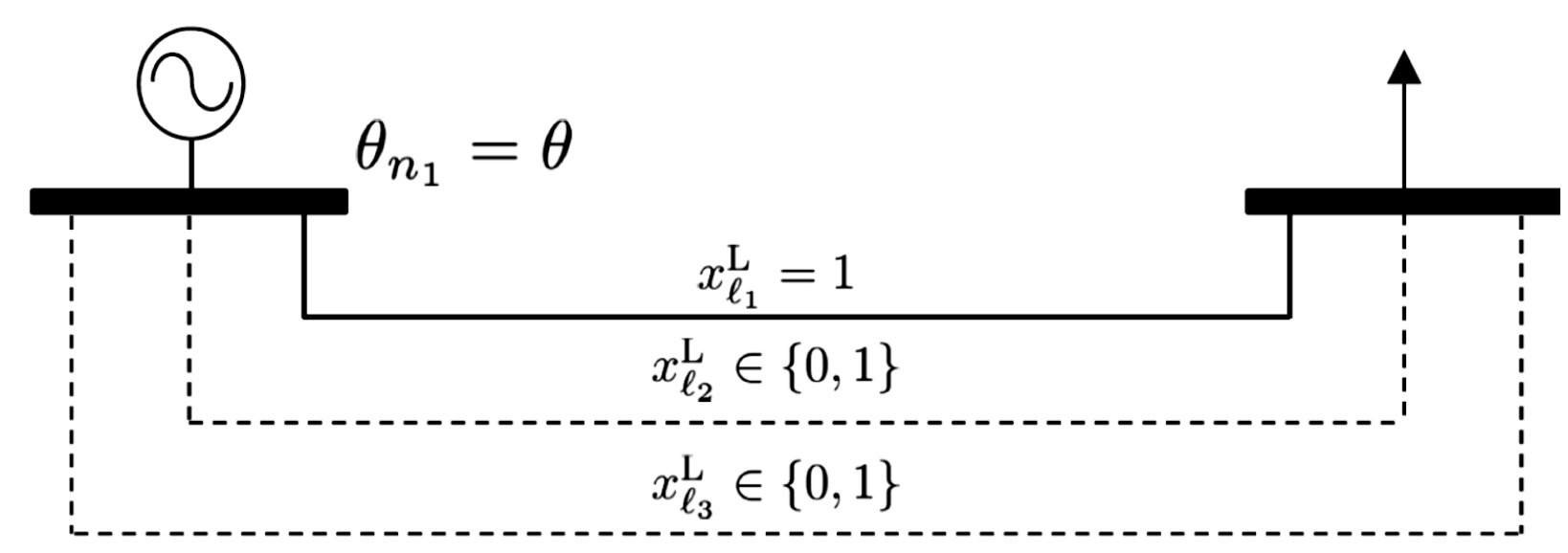




\section{Transmission Expansion Planning}

Considering the above data results in the following optimization problem: 
$\min _{x_{\ell_{2}}^{\mathrm{L}}, x_{\ell_{3}}^{\mathrm{L}}, p^{\mathrm{G}}, \theta} \quad I x_{\ell_{2}}^{\mathrm{L}}+I x_{\ell_{3}}^{\mathrm{L}}+\sigma C p^{\mathrm{E}}$

s.t.

$$
\begin{aligned}
& x_{\ell_{2}}^{\mathrm{L}}, x_{\ell_{3}}^{\mathrm{L}}=\{0,1\} \\
& p^{\mathrm{E}}=p_{\ell_{1}}^{\mathrm{L}}+p_{\ell_{2}}^{\mathrm{L}}+p_{\ell_{3}}^{\mathrm{L}} \\
& p_{\ell_{1}}^{\mathrm{L}}+p_{\ell_{2}}^{\mathrm{L}}+p_{\ell_{3}}^{\mathrm{L}}=P^{\mathrm{D}^{\max }} \\
& p_{\ell_{1}}^{\mathrm{L}}=\theta \\
& p_{\ell_{2}}^{\mathrm{L}}=x_{\ell_{2}}^{\mathrm{L}} \theta \\
& p_{\ell_{3}}^{\mathrm{L}}=x_{\ell_{3}}^{\mathrm{L}} \theta \\
& -F^{\max } \leq p_{\ell_{1}}^{\mathrm{L}} \leq F^{\max } \\
& -F^{\max } \leq p_{\ell_{2}}^{\mathrm{L}} \leq F^{\max } \\
& -F^{\max } \leq p_{\ell_{3}}^{\mathrm{L}} \leq F^{\max } \\
& 0 \leq p^{\mathrm{E}} \leq \infty \\
& -\pi \leq \theta \leq \pi
\end{aligned}
$$

\section{Transmission}

\section{Expansion Planning}

$P^{\mathrm{D}^{\max }}$

$\theta_{n_{2}}=0$ 


\section{Transmission Expansion Planning}

If $0 \leq P^{D^{\max }} \leq F^{\max }$, the capacity of the existing transmission line allows the power flow from the generating unit at node 1 to the demand at node 2 . No need to build any additional transmission line.

If $P^{\mathrm{D}^{\max }}>F^{\max }$, the capacity of the existing transmission line $\ell_{1}$ is not enough. 


\section{Transmission Expansion Planning}

If $F^{\max }<P^{D^{\max }} \leq 2 F^{\max }$, then it is necessary to build one additional line between nodes 1 and 2 .

If $2 F^{\max }<P^{\mathrm{D}^{\max }} \leq 3 F^{\max }$, then we need to build two additional lines.

If $P^{D^{\max }}>3 F^{\max }$, the problem is infeasible and additional expansion options must be considered. 


\section{Transmission Expansion Planning}

The optimal solution of this illustrative example depends on the value of $P^{\mathrm{D}^{\max }}$.

Indeed, we build zero, one, or two additional lines depending on the value of the expected largest demand in the planning horizon. 


\section{Transmission Expansion Planning - Example II}

In the previous example, we solve the TEP problem considering a static approach (investment + operation).

Let us now consider that this planning horizon is divided in two periods, $t_{1}$ and $t_{2}$.

The expected largest demands in these two time periods are $P_{t_{1}}^{\mathrm{D}^{\max }}$ and $P_{t_{2}}^{\mathrm{D}^{\max }}$, respectively. 


\section{Transmission Expansion Planning - Example II}

Instead of a static approach, we consider that the transmission planner can build lines at the beginning of each of the two considered periods, i.e., we consider a dynamic approach.

The figure below illustrates the TEP problem in this case. 


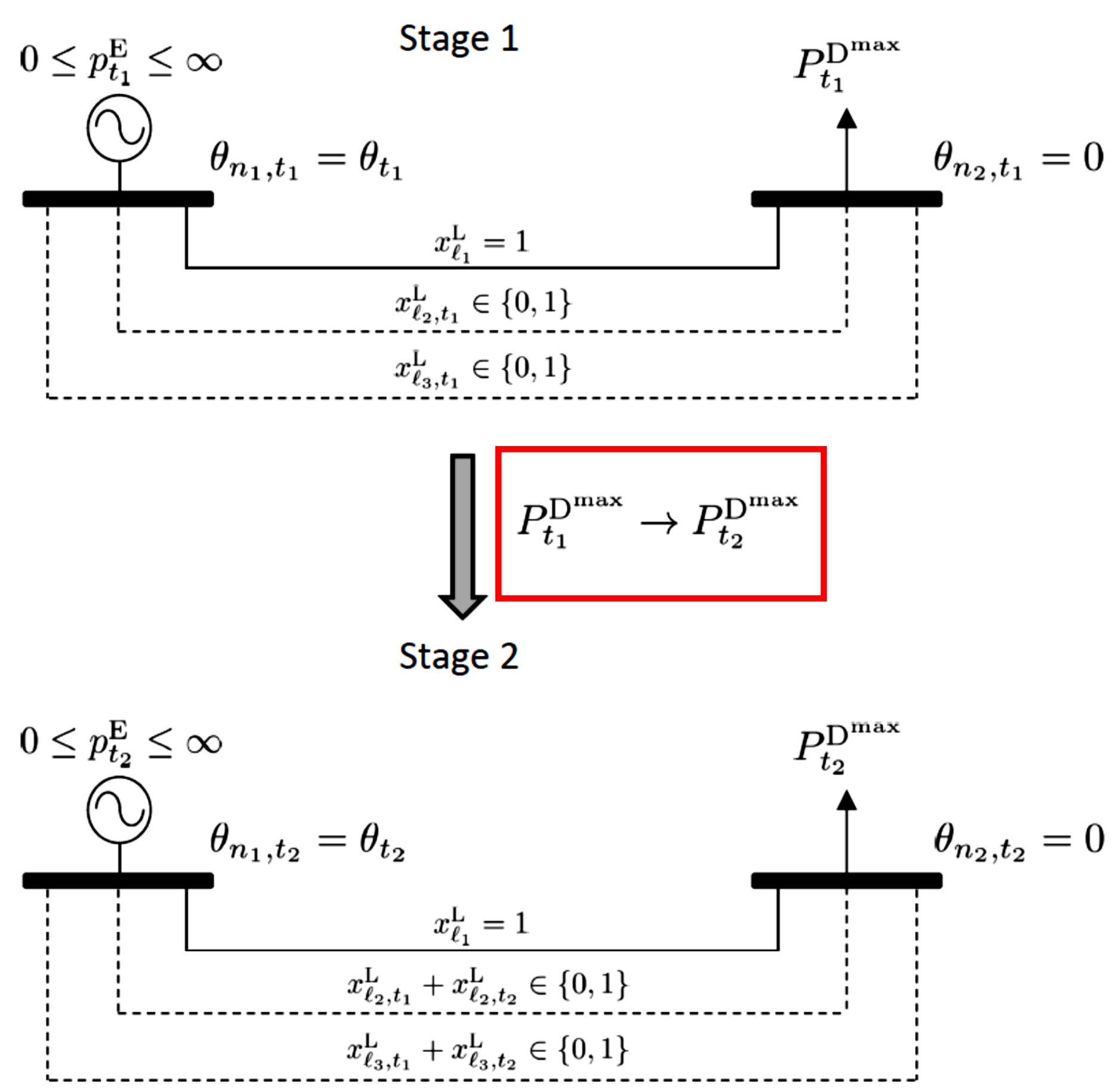




\section{Transmission Expansion Planning}

Considering the above data, the TEP problem considering a dynamic approach results in the following optimization problem: 


$$
\begin{gathered}
\min _{x_{\ell_{2} t_{1}}^{\mathrm{L}}, x_{\ell_{3} t_{1}}^{\mathrm{L}}, x_{\ell_{2} t_{2}}^{\mathrm{L}}, x_{\ell_{3} t_{2}}^{\mathrm{L}}, p_{t_{1}}^{\mathrm{G}}, p_{t_{2}}^{\mathrm{G}}, \theta_{t_{1}}, \theta_{t_{2}}} 2 I x_{\ell_{2} t_{1}}^{\mathrm{L}}+2 I x_{\ell_{3} t_{1}}^{\mathrm{L}}+I x_{\ell_{2} t_{2}}^{\mathrm{L}}+I x_{\ell_{3} t_{2}}^{\mathrm{L}} \\
+\sigma C\left(p_{t_{1}}^{\mathrm{E}}+p_{t_{2}}^{\mathrm{E}}\right)
\end{gathered}
$$

subject to
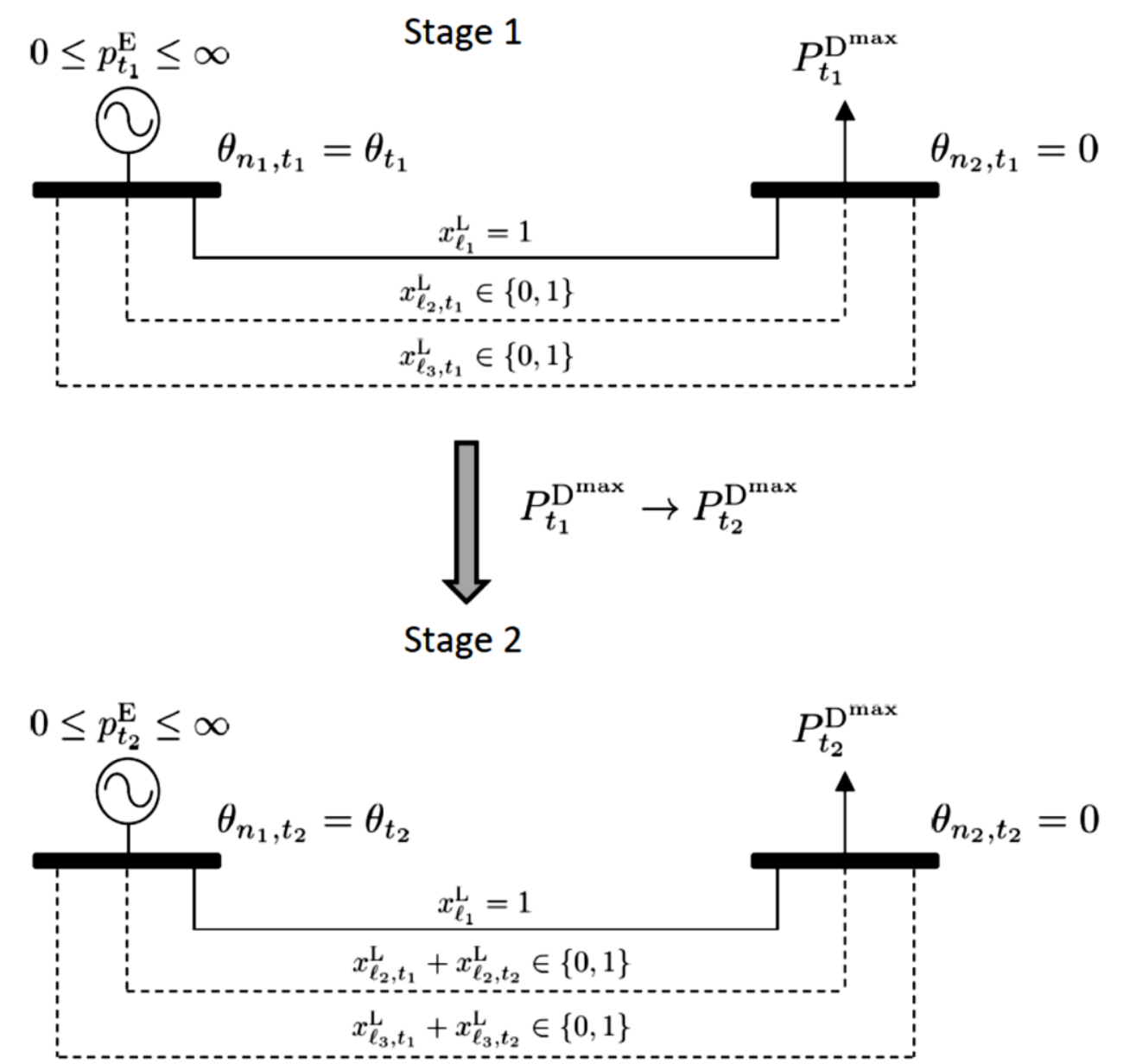

$$
\begin{aligned}
& x_{\ell_{2} t_{1}}^{\mathrm{L}}, x_{\ell_{3} t_{1}}^{\mathrm{L}}, x_{\ell_{2} t_{2}}^{\mathrm{L}}, x_{\ell_{3} t_{2}}^{\mathrm{L}}=\{0,1\} \\
& x_{\ell_{2} t_{1}}^{\mathrm{L}}+x_{\ell_{2} t_{2}}^{\mathrm{L}} \leq 1 \\
& x_{\ell_{3} t_{1}}^{\mathrm{L}}+x_{\ell_{3} t_{2}}^{\mathrm{L}} \leq 1 \\
& p_{t_{1}}^{\mathrm{E}}-p_{\ell_{1} t_{1}}^{\mathrm{L}}-p_{\ell_{2} t_{1}}^{\mathrm{L}}-p_{\ell_{3} t_{1}}^{\mathrm{L}}=0 \\
& p_{\ell_{1} t_{1}}^{\mathrm{L}}+p_{\ell_{2} t_{1}}^{\mathrm{L}}+p_{\ell_{3} t_{1}}^{\mathrm{L}}=P_{t_{1}}^{\mathrm{D}^{\max }} \\
& p_{\ell_{1} t_{1}}^{\mathrm{L}}=\theta_{t_{1}} \\
& p_{\ell_{2} t_{1}}^{\mathrm{L}}=x_{\ell_{2} t_{1}}^{\mathrm{L}} \theta_{t_{1}} \\
& p_{\ell_{3} t_{1}}^{\mathrm{L}}=x_{\ell_{3} t_{1}}^{\mathrm{L}} \theta_{t_{1}}
\end{aligned}
$$




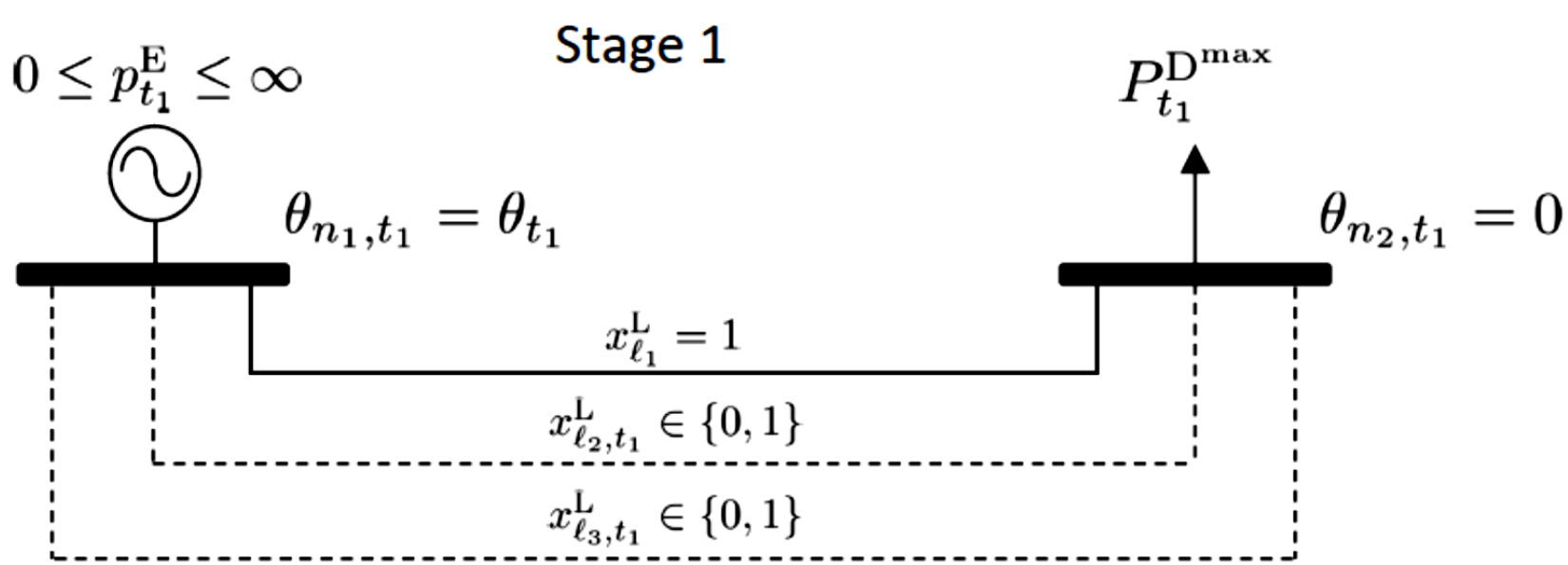

$$
\prod P_{t_{1}}^{\mathrm{D}^{\max }} \rightarrow P_{t_{2}}^{\mathrm{D}^{\max }}
$$

\section{Stage 2}

$0 \leq p_{t_{2}}^{\mathrm{E}} \leq \infty$

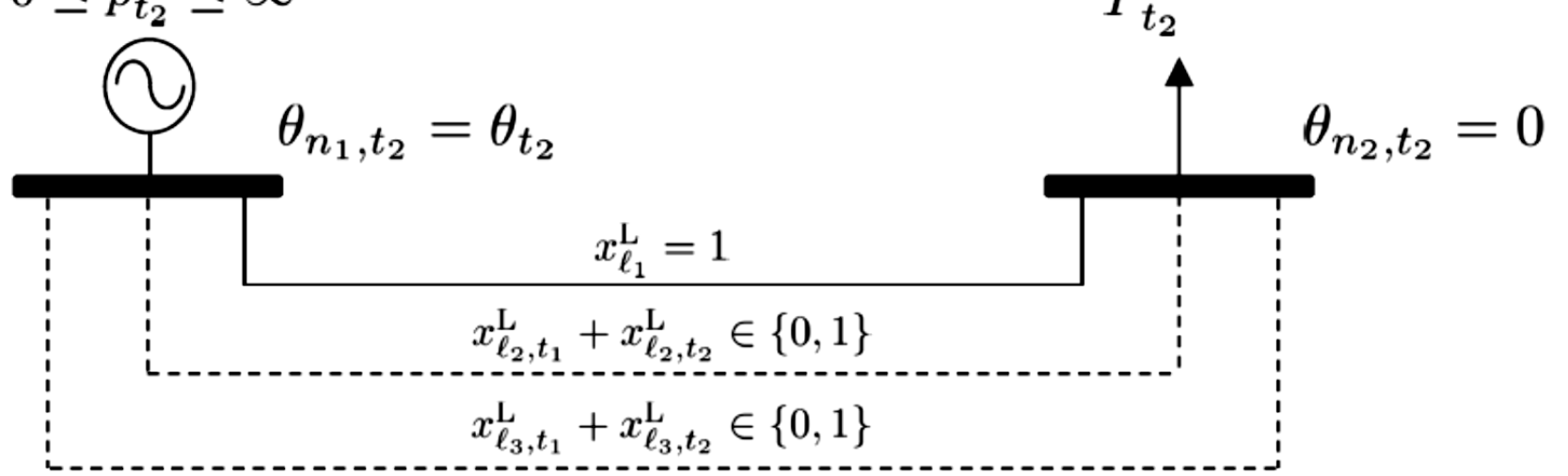

$$
\begin{aligned}
& -F^{\max } \leq p_{\ell_{1} t_{1}}^{\mathrm{L}} \leq F^{\max } \\
& -F^{\max } \leq p_{\ell_{2} t_{1}}^{\mathrm{L}} \leq F^{\max } \\
& -F^{\max } \leq p_{\ell_{3} t_{1}}^{\mathrm{L}} \leq F^{\max } \\
& 0 \leq p_{t_{1}}^{\mathrm{E}} \leq \infty \\
& -\pi \leq \theta_{t_{1}} \leq \pi \\
& p_{t_{2}}^{\mathrm{E}}-p_{\ell_{1} t_{2}}^{\mathrm{L}}-p_{\ell_{2} t_{2}}^{\mathrm{L}}-p_{\ell_{3} t_{2}}^{\mathrm{L}}=0 \\
& p_{\ell_{1} t_{2}}^{\mathrm{L}}+p_{\ell_{2} t_{2}}^{\mathrm{L}}+p_{\ell_{3} t_{2}}^{\mathrm{L}}=P_{t_{2}}^{\mathrm{D}^{\max }} \\
& p_{\ell_{1} t_{2}}^{\mathrm{L}}=\theta_{t_{2}} \\
& p_{\ell_{2} t_{2}}^{\mathrm{L}}=\left(x_{\ell_{2} t_{1}}^{\mathrm{L}}+x_{\ell_{2} t_{2}}^{\mathrm{L}}\right) \theta_{t_{2}} \\
& p_{\ell_{3} t_{2}}^{\mathrm{L}}=\left(x_{\ell_{3} t_{1}}^{\mathrm{L}}+x_{\ell_{3} t_{2}}^{\mathrm{L}}\right) \theta_{t_{2}} \\
& -F^{\max } \leq p_{\ell_{1} t_{2}}^{\mathrm{L}} \leq F^{\max } \\
& -F^{\max } \leq p_{\ell_{2} t_{2}}^{\mathrm{L}} \leq F^{\max } \\
& -F^{\max } \leq p_{\ell_{3} t_{2}}^{\mathrm{L}} \leq F^{\max } \\
& 0 \leq p_{t_{2}}^{\mathrm{E}} \leq \infty \\
& -\pi \leq \theta_{t_{2}} \leq \pi
\end{aligned}
$$




\section{Transmission Expansion Planning}

Differences between this model and the previous one are:

1. Prospective lines built in the first period remain built in the second period.

2. The investment cost of a line built in the first period is twice that of a line built in the second one since it is used twice.

3. Constraints are considered for both time periods.

4. Subindexes $t_{1}$ and $t_{2}$ identify time periods 1 and 2 , respectively. 


\section{Transmission Expansion Planning}

As in the previous example, the optimal transmission expansion plan depends on the value of $P_{t_{1}}^{\mathrm{D}^{\max }}$ and $P_{t_{2}}^{\mathrm{D}^{\max }}$ :

1. In the first time period:

- If $0 \leq P_{t_{1}}^{\mathrm{D}^{\max }} \leq F^{\max }$, we build no line.

- If $F^{\max }<P_{t_{1}}^{\mathrm{D}^{\max }} \leq 2 F^{\max }$, we build one line.

- If $2 F^{\max }<P_{t_{1}}^{\mathrm{D}^{\max }} \leq 3 F^{\max }$, we build two lines. 


\section{Transmission Expansion Planning}

2. In the second period, the expansion plan depends on $P_{t_{2}}^{\mathrm{D}^{\max }}$ and also on the expansion plan in the first period.

Assuming that $P_{t_{2}}^{\mathrm{D}^{\max }} \geq P_{t_{1}}^{\mathrm{D}^{\max }}$, the transmission expansion plan in the second period is as follows: 


\section{Transmission Expansion Planning}

- If no line is built in the first time period, we build no line if $0 \leq P_{t_{2}}^{\mathrm{D}^{\max }} \leq F^{\max }$, one line if $F^{\max }<P_{t_{2}}^{\mathrm{D}^{\max }} \leq 2 F^{\max }$, and two lines if $2 F^{\max }<P_{t_{2}}^{\mathrm{D}^{\max }} \leq 3 F^{\max }$.

- If one line is built in the first period, we build no line if $F^{\max }<P_{t_{2}}^{\mathrm{D}^{\max }} \leq 2 F^{\max }$ and one line if $2 F^{\max }<P_{t_{2}}^{\mathrm{D}^{\max }} \leq 3 F^{\max }$.

- If two lines are built in the first time period, no line is built. 


\section{Transmission Expansion Planning}

3. If $P_{t_{1}}^{\mathrm{D}^{\max }}>3 F^{\max }$ or $P_{t_{2}}^{\mathrm{D}^{\max }}>3 F^{\max }$, the problem is infeasible, and additional expansion options should be considered. 


\section{Transmission Expansion Planning}

This example is comparatively more complex than the previous one since the number of binary variables and constraints is approximately double.

However, with a dynamic approach we can adapt to future changes, and therefore, the solution is usually superior to that obtained with a static approach. 


\section{Transmission Expansion Planning}

These examples are simple and their solutions trivial.

However, as the size of the system and the number of prospective transmission lines increase, the TEP problem becomes complex and its solution is no longer trivial. 


\section{Transmission Expansion Planning}

These formulations include binary variables, and products of binary and continuous variables, i.e., they are MINLP problems.

These problems are usually hard to solve. Nevertheless, it is possible to remove the non-linearities. 


\section{Transmission Expansion Planning - Static Formulation}

Notation 


\section{Transmission Expansion Planning}

Indices:

$d$ Demands.

$g$ Generating units.

$\ell$ Transmission lines.

$n$ Nodes. 


\section{Transmission Expansion Planning}

Sets:

$r(\ell)$ Receiving-end node of transmission line $\ell$.

$s(\ell)$ Sending-end node of transmission line $\ell$.

$\Omega_{n}^{\mathrm{D}}$ Demands located at node $n$.

$\Omega_{n}^{\mathrm{E}}$ Generating units located at node $n$.

$\Omega^{\mathrm{L}+}$ Prospective transmission lines. 


\section{Transmission Expansion Planning}

Parameters:

$B_{\ell}$ Susceptance of transmission line $\ell[S]$.

$C_{d}^{\mathrm{LS}}$ Load-shedding cost of demand $d[\$ / \mathrm{MWh}]$.

$C_{g}^{\mathrm{E}}$ Production cost of generating unit $g[\$ / \mathrm{MWh}]$.

$F_{\ell}^{\max }$ Capacity of transmission line $\ell[\mathrm{MW}]$. 


\section{Transmission Expansion Planning}

Parameters:

$\tilde{I}_{\ell}^{\mathrm{L}}$ Annualized investment cost of line $\ell[\$ / \mathrm{MW}]$.

$\tilde{I}^{\mathrm{L}, \max }$ Annualized investment budget for building lines [\$].

$P_{d}^{\mathrm{D}^{\max }}$ Load of demand $d[\mathrm{MW}]$.

$P_{g}^{\mathrm{E}^{\max }}$ Capacity of generating unit $g[\mathrm{MW}]$. 


\title{
Transmission Expansion Planning
}

\author{
Binary Variables: \\ $x_{\ell}^{\mathrm{L}}$ Binary variable that is equal to 1 if prospective trans- \\ mission line $\ell$ is built and 0 otherwise.
}




\section{Transmission Expansion Planning}

Continuous Variables:

$p_{g}^{\mathrm{E}}$ Power produced by generating unit $g[\mathrm{MW}]$.

$p_{\ell}^{\mathrm{L}}$ Power flow through transmission line $\ell$ [MW].

$p_{d}^{\mathrm{LS}}$ Load shed by demand $d[\mathrm{MW}]$.

$\theta_{n}$ Voltage angle at node $n[\mathrm{rad}]$. 


\title{
Transmission Expansion Planning
}

\author{
Deterministic Static Approach
}




$$
\min _{\Delta} \quad \sum_{\ell \in \Omega^{\mathrm{L}+}} \tilde{I}_{\ell}^{\mathrm{L}} x_{\ell}^{\mathrm{L}}+\sigma\left[\sum_{g} C_{g}^{\mathrm{E}} p_{g}^{\mathrm{E}}+\sum_{d} C_{d}^{\mathrm{LS}} p_{d}^{\mathrm{LS}}\right]
$$

subject to

$$
\begin{aligned}
& \sum_{\ell \in \Omega^{\mathrm{L}}+} \tilde{I}_{\ell}^{\mathrm{L}} x_{\ell}^{\mathrm{L}} \leq \tilde{I}^{\mathrm{L}, \max } \\
& x_{\ell}^{\mathrm{L}}=\{0,1\} \quad \forall \ell \in \Omega^{\mathrm{L}+}
\end{aligned}
$$

Transmission

$\sum_{g \in \Omega_{n}^{\mathrm{E}}} p_{g}^{\mathrm{E}}-\sum_{\ell \mid s(\ell)=n} p_{\ell}^{\mathrm{L}}+\sum_{\ell \mid r(\ell)=n} p_{\ell}^{\mathrm{L}}=\sum_{d \in \Omega_{n}^{\mathrm{D}}}\left(P_{d}^{\mathrm{D}^{\max }}-p_{d}^{\mathrm{LS}}\right) \quad \forall n$

Expansion

Planning

$$
\begin{aligned}
& p_{l}^{\mathrm{L}}=B_{\ell}\left(\theta_{s(\ell)}-\theta_{r(\ell)}\right) \quad \forall \ell \backslash \ell \in \Omega^{\mathrm{L}+} \\
& p_{l}^{\mathrm{L}}=x_{\ell}^{\mathrm{L}} B_{\ell}\left(\theta_{s(\ell)}-\theta_{r(\ell)}\right) \quad \forall \ell \in \Omega^{\mathrm{L}+} \\
& -F_{\ell}^{\max } \leq p_{\ell}^{\mathrm{L}} \leq F_{\ell}^{\max } \quad \forall \ell \\
& 0 \leq p_{g}^{\mathrm{E}} \leq P_{g}^{\mathrm{E}^{\max }} \quad \forall g \\
& 0 \leq p_{d}^{\mathrm{LS}} \leq P_{d}^{\mathrm{D}^{\max }} \quad \forall d \\
& -\pi \leq \theta_{n} \leq \pi \quad \forall n \\
& \theta_{n}=0 \quad n \text { : reference }
\end{aligned}
$$




\section{Transmission Expansion Planning}

where variables in set

$$
\Delta=\left\{x_{\ell}^{\mathrm{L}}, p_{\ell}^{\mathrm{L}}, p_{g}^{\mathrm{G}}, p_{d}^{\mathrm{D}}, \theta_{n}\right\}
$$

are the optimization variables of problem (1). 


\section{Transmission Expansion Planning}

Objective function (1a) comprises the three terms below:

1. $\sum_{\ell \in \Omega^{\mathrm{L}+}} I_{\ell}^{\mathrm{L}} x_{\ell}^{\mathrm{L}}$ is the annualized cost of building new lines.

2. $\sum_{g} C_{g}^{\mathrm{E}} p_{g}^{\mathrm{E}}$ is the operating cost of generating units.

3. $\sum_{d} C_{d}^{\mathrm{LS}} p_{d}^{\mathrm{LS}}$ is the load-shedding cost. 


\section{Transmission Expansion Planning}

Terms in 2 and 3 above are multiplied by the factor $\sigma$ to make operation and investment costs comparable.

Since we consider that $\tilde{I}_{\ell}^{\mathrm{L}}$ are annualized investment costs, then $\sigma$ is equal to 8760 h, i.e., the total number of hours in a year. 


\section{Transmission Expansion Planning}

Constraint (1b) ensures that the cost of building new lines is below the available budget.

Constraints (1c) define binary variables $x_{\ell}^{\mathrm{L}}$ that indicate whether a line is built $\left(x_{\ell}^{\mathrm{L}}=1\right)$ or not $\left(x_{\ell}^{\mathrm{L}}=0\right)$.

Constraints (1d) impose the generation-demand balance at each node. 


\section{Transmission Expansion Planning}

Equations (1e) and (1f) define the power flows through all lines, which are bounded by constraints (1g).

Subindexes $s(\ell)$ and $r(\ell)$ denote the sending-end and receiving-end nodes of transmission line $\ell$, respectively. 


\section{Transmission Expansion Planning}

Constraints (1h) and (1i) bound the power produced by units and the unserved demand, respectively.

Constraints $(1 \mathrm{j})$ and $(1 \mathrm{k})$ bound voltage angles and fix to zero the voltage angle at the reference node, respectively. 


\section{TEP - Uncertainty}




\section{TEP - Uncertainty}

Two-Stage Stochastic Programming 


\section{TEP - Uncertainty - Example}

A transmission planner has the option of building just 1 of 2 alternative transmission lines of capacity either 2 or 4 to connect nodes 1 and 2 .

The per unit building cost for the line is 1 .

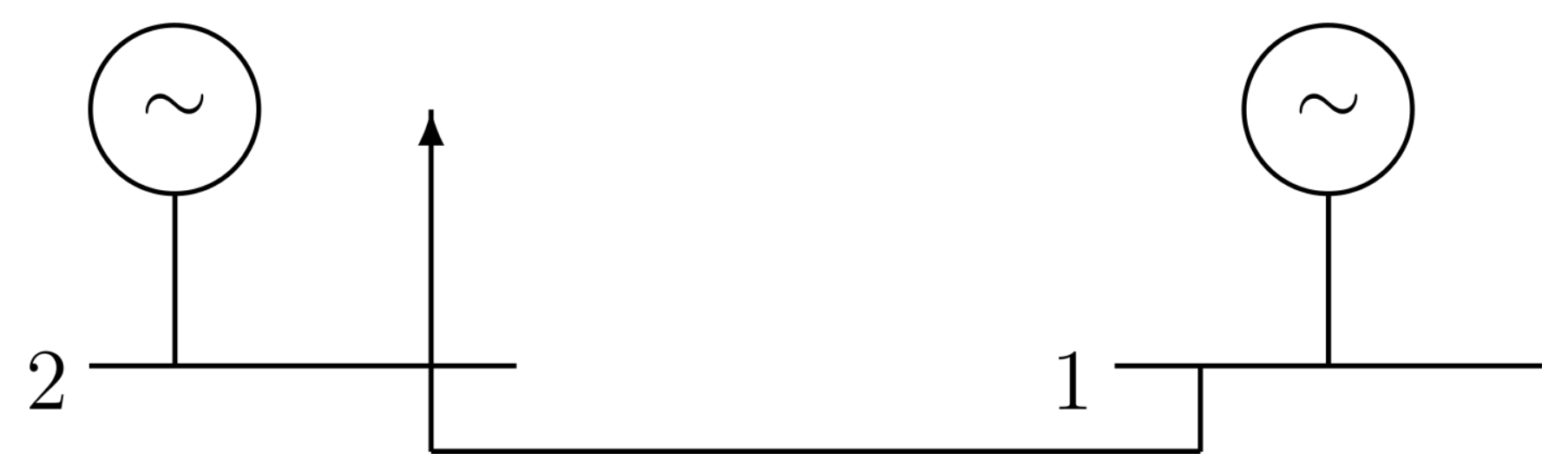




\section{TEP - Uncertainty}
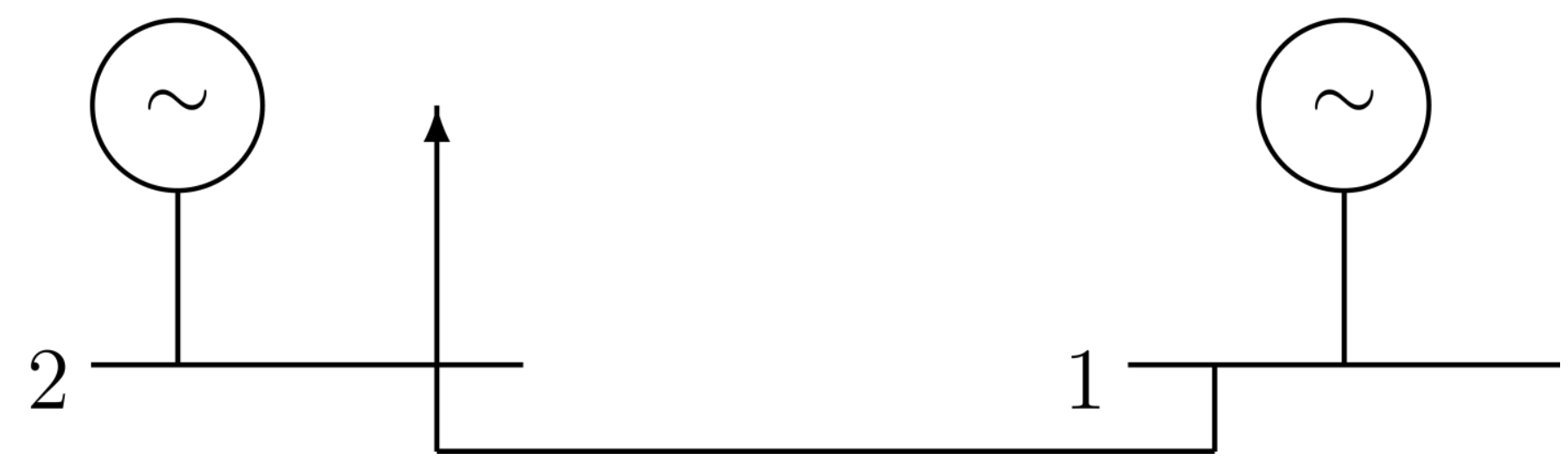

At node 1, a generator of unlimited capacity and per unit production cost of 5 is available, while

at node 2 another generator also of unlimited capacity and per unit production cost of 10 is available. 


\section{TEP - Uncertainty}

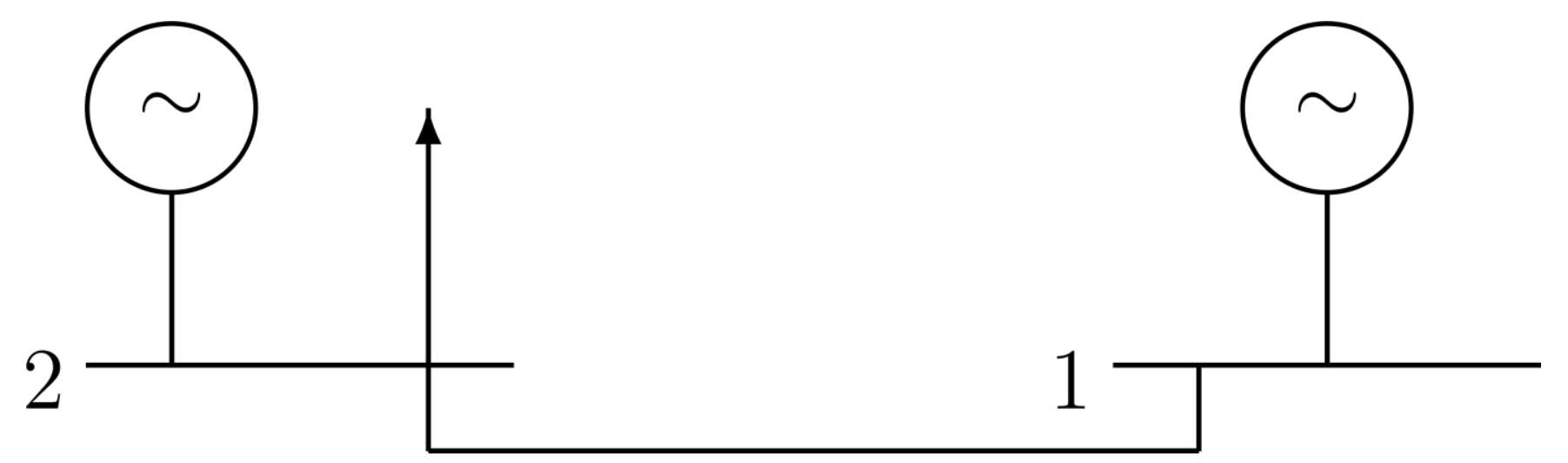

The demand is located at node 2 and can take only two values, either 3 or 5 , with equal probability, $\frac{1}{2}$.

We note that the demand uncertainty is conveniently visualized using a scenario tree, as shown in the figure below. 


\section{TEP - Uncertainty}

The demand is located at node 2 and can take only two values, either 3 or 5 , with equal probability, $\frac{1}{2}$.

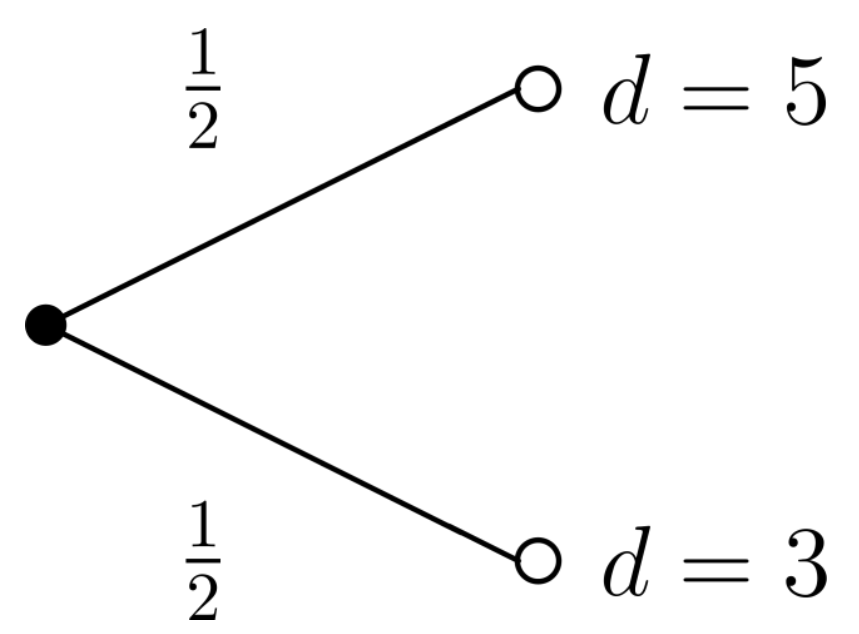




\section{TEP - Uncertainty}

A two-stage SP model for the transmission planner to decide which transmission line to build is: 
TEP - Uncertainty

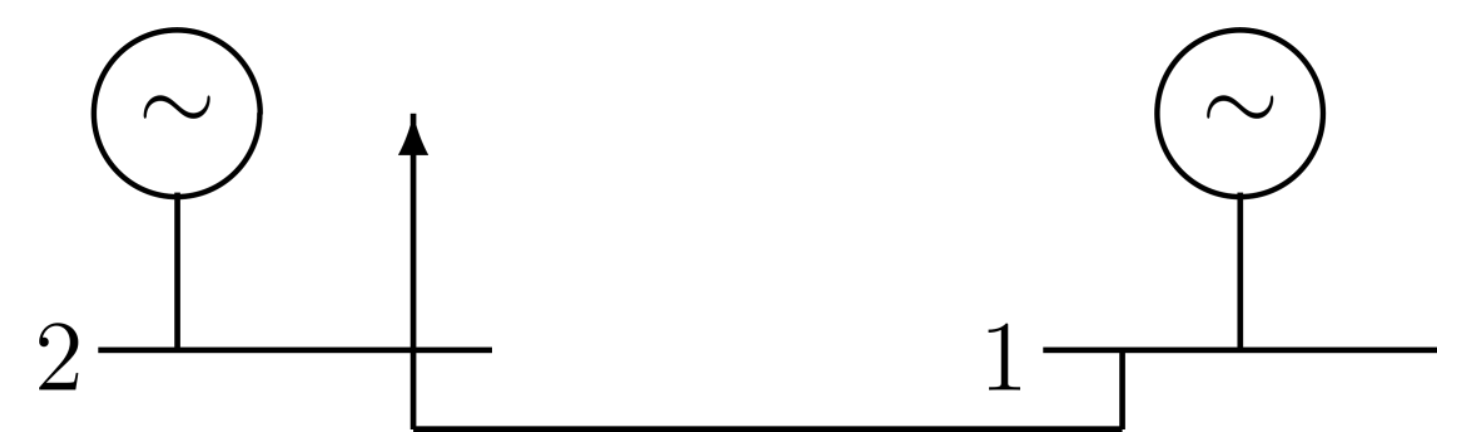

$$
\begin{array}{rl|}
\min _{x \in\{2,4\}}\left[1 x+\min _{y_{1}, y_{2} \geq 0}\right. & \frac{1}{2}\left(5 y_{1}^{(1)}+10 y_{2}^{(1)}\right)+\frac{1}{2}\left(5 y_{1}^{(2)}+10 y_{2}^{(2)}\right) \\
\text { s.t. } & \begin{array}{l}
y_{1}^{(1)}+y_{2}^{(1)}=3 \\
y_{1}^{(1)} \leq x
\end{array}
\end{array}
$$

$$
\begin{aligned}
& y_{1}^{(2)}+y_{2}^{(2)}=5 \\
& y_{1}^{(2)} \leq x
\end{aligned}
$$




\section{TEP - Uncertainty}

$x$ is the capacity of the transmission line to be built,

$y_{1}^{(1)}$ and $y_{2}^{(1)}$ the production of the units at nodes 1 and 2 , respectively, if the demand is $d=3$ (scenario 1 identified by superscript (1)), and

$y_{1}^{(2)}$ and $y_{2}^{(2)}$ the production of the units at nodes 1 and 2 , respectively, if the demand is $d=5$ (scenario 2 identified by superscript (2)). 


$$
\begin{aligned}
& \min _{x \in\{2,4\}} 1 x+\min _{y_{1}, y_{2} \geq 0} \frac{1}{2}\left(5 y_{1}^{(1)}+10 y_{2}^{(1)}\right)+\frac{1}{2}\left(5 y_{1}^{(2)}+10 y_{2}^{(2)}\right) \\
& \text { s.t. } \quad y_{1}^{(1)}+y_{2}^{(1)}=3 \\
& y_{1}^{(1)} \leq x \\
& \text { TEP - Uncertainty } \\
& y_{1}^{(2)}+y_{2}^{(2)}=5 \\
& y_{1}^{(2)} \leq x
\end{aligned}
$$

The first term of the objective function of the problem above corresponds to the investment cost in the line, while

the second one corresponds to the expected operation cost over the two possible scenarios, each one involving a weight of $\frac{1}{2}$. 


$$
\begin{aligned}
& \min _{x \in\{2,4\}} 1 x+\min _{y_{1}, y_{2} \geq 0} \frac{1}{2}\left(5 y_{1}^{(1)}+10 y_{2}^{(1)}\right)+\frac{1}{2}\left(5 y_{1}^{(2)}+10 y_{2}^{(2)}\right) \\
& \text { s.t. } \quad y_{1}^{(1)}+y_{2}^{(1)}=3 \\
& y_{1}^{(1)} \leq x \\
& y_{1}^{(2)}+y_{2}^{(2)}=5 \\
& \text { TEP - Uncertainty } \\
& y_{1}^{(2)} \leq x
\end{aligned}
$$

Constraints pertain to the actual operation of the power system in both scenarios, and include energy balance and transmission limit. 


\section{TEP - Uncertainty}

The problem above is equivalent to: 


$$
\begin{array}{lll}
\min _{x \in\{2,4\}} 1 x+\min _{y_{1}, y_{2} \geq 0} & \frac{1}{2}\left(5 y_{1}^{(1)}+10 y_{2}^{(1)}\right)+\frac{1}{2}\left(5 y_{1}^{(2)}+10 y_{2}^{(2)}\right) \\
& \text { s.t. } & y_{1}^{(1)}+y_{2}^{(1)}=3 \\
& y_{1}^{(1)} \leq x \\
& y_{1}^{(2)}+y_{2}^{(2)}=5 \\
& y_{1}^{(2)} \leq x
\end{array}
$$

\section{$\min _{x \in\{2,4}$}

TEP - Uncertainty

$$
\begin{array}{ll}
\min _{x \in\{2,4\} ; y_{1}, y_{2} \geq 0} & z=1 x+\frac{1}{2}\left(5 y_{1}^{(1)}+10 y_{2}^{(2)}\right)+\frac{1}{2}\left(5 y_{1}^{(1)}+10 y_{2}^{(2)}\right) \\
\text { s.t. } & \\
& y_{1}^{(1)}+y_{2}^{(1)}=3 \\
& y_{1}^{(1)} \leq x \\
& y_{1}^{(2)}+y_{2}^{(2)}=5 \\
& y_{1}^{(2)} \leq x
\end{array}
$$




\section{TEP - Uncertainty}

Given the small size and simplicity of this problem, it can be solved by enumeration.

This is done below. 


\section{TEP - Uncertainty}

We first consider that the planner builds the line of capacity 2, i.e., $x=2$. Then, the problem to be solved is:

$$
\begin{array}{ll}
\min _{y_{1}, y_{2} \geq 0} & z=2+\frac{1}{2}\left(5 y_{1}^{(1)}+10 y_{2}^{(1)}\right)+\frac{1}{2}\left(5 y_{1}^{(2)}+10 y_{2}^{(2)}\right) \\
\text { s.t. } & y_{1}^{(1)}+y_{2}^{(1)}=3 \\
& y_{1}^{(1)} \leq 2 \\
& y_{1}^{(2)}+y_{2}^{(2)}=5 \\
& y_{1}^{(2)} \leq 2,
\end{array}
$$

whose solution can be easily obtained and is $y_{1}^{(1)}=2, y_{2}^{(1)}=1$, $y_{1}^{(2)}=2, y_{2}^{(2)}=3$, and $z=32$. 


\section{TEP - Uncertainty}

We then consider that the planner builds the line of capacity 4 , i.e., $x=4$. Then, the problem to be solved is:

$$
\begin{array}{ll}
\min _{y_{1}, y_{2} \geq 0} & z=4+\frac{1}{2}\left(5 y_{1}^{(1)}+10 y_{2}^{(1)}\right)+\frac{1}{2}\left(5 y_{1}^{(2)}+10 y_{2}^{(2)}\right) \\
\text { s.t. } & y_{1}^{(1)}+y_{2}^{(1)}=3 \\
& y_{1}^{(1)} \leq 4 \\
& y_{1}^{(2)}+y_{2}^{(2)}=5 \\
& y_{1}^{(2)} \leq 4,
\end{array}
$$

whose solution is $y_{1}^{(1)}=3, y_{2}^{(1)}=0, y_{1}^{(2)}=4, y_{2}^{(2)}=1$, and $z=26.5$. 


\section{TEP - Uncertainty}

Considering the two alternative for investment $x=2$ $(z=32)$ and $x=4(z=26.5)$, and the fact that the planner seeks minimum total cost, the solution is

$$
x=4, y_{1}^{(1)}=3, y_{2}^{(1)}=0, y_{1}^{(2)}=4, y_{2}^{(2)}=1 \text {, and } z=26.5 \text {. }
$$

The planner builds the larger line $(x=4)$ because this is best on average. 


\section{TEP - Uncertainty}

A two-stage stochastic programming problem has the form below:

$$
\min _{\mathbf{x}^{1} \in \mathcal{X}^{1}} f^{1}\left(\mathbf{x}^{1}\right)+\mathbb{E}_{\xi^{1}}\left\{z_{\xi^{1}}^{1}\right\}
$$

where:

$$
z_{\xi^{1}}^{1}=\left\{\min _{\mathbf{x}_{\xi^{1}}^{2} \in \mathcal{X}^{2}\left(\xi^{1}\right)} f^{2}\left(\mathbf{x}^{1}, \mathbf{x}_{\xi^{1}}^{2}\right)\right\}
$$


$\mathbf{x}^{1} \in \mathcal{X}^{1}$ is the vector of first-stage optimization variables within the feasible set $\mathcal{X}^{1}$,

$f^{1}\left(\mathbf{x}^{1}\right)$ is the part of the objective function related to the vector of first-stage optimization variables,

$\mathbb{E}_{\xi^{1}}\left\{z_{\xi^{1}}^{1}\right\}$ is the expected value of the part of the objective function specifically related to the vector of second-stage optimization variables. 


\section{TEP - Uncertainty}

$$
z_{\xi^{1}}^{1}=\left\{\min _{\mathbf{x}_{\xi^{1}}^{2} \in \mathcal{X}^{2}\left(\xi^{1}\right)} f^{2}\left(\mathbf{x}^{1}, \mathbf{x}_{\xi^{1}}^{2}\right)\right\}
$$

$\mathbf{x}_{\xi^{1}}^{2}$ is the vector of second-stage optimization variables within the feasible set $\mathcal{X}^{2}\left(\xi^{1}\right)$, which take optimal values once the uncertain parameters have taken specific values (and the uncertainty has thus vanished),

$f^{2}\left(\mathbf{x}^{1}, \mathbf{x}_{\xi^{1}}^{2}\right)$ is the part of the objective function related to the vector of second-stage optimization variables. 
TEP - Uncertainty

Adaptive Robust Optimization (ARO) 


\section{TEP - Uncertainty - Example}

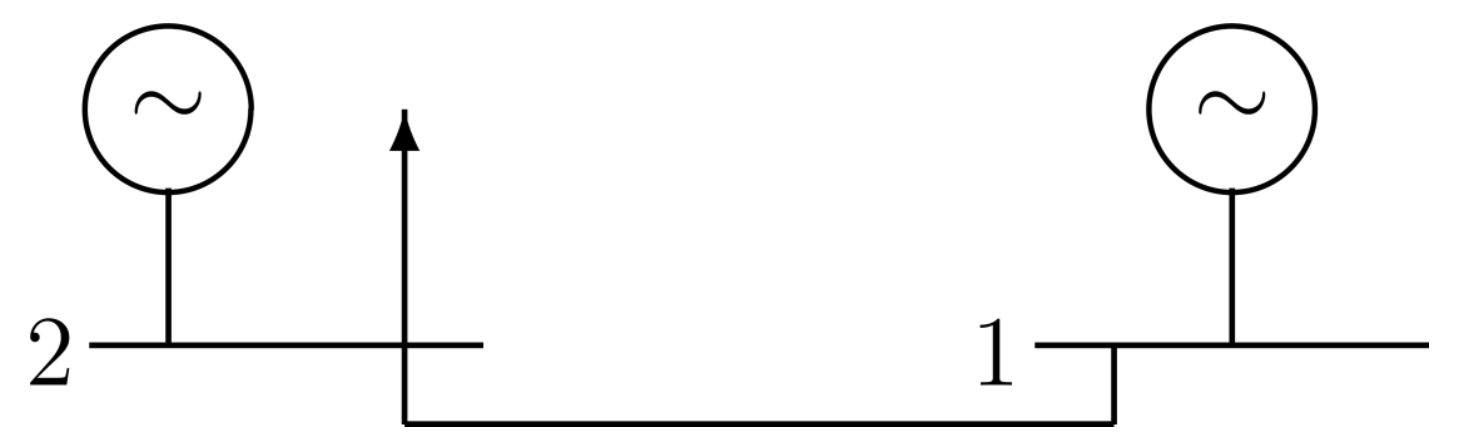

We recall the transmission expansion planner that has the option of building just 1 of 2 alternatives lines of capacity either 2 or 4 to connect nodes 1 and 2 .

The per unit building cost for the lines is 1 . 


\section{TEP - Uncertainty}

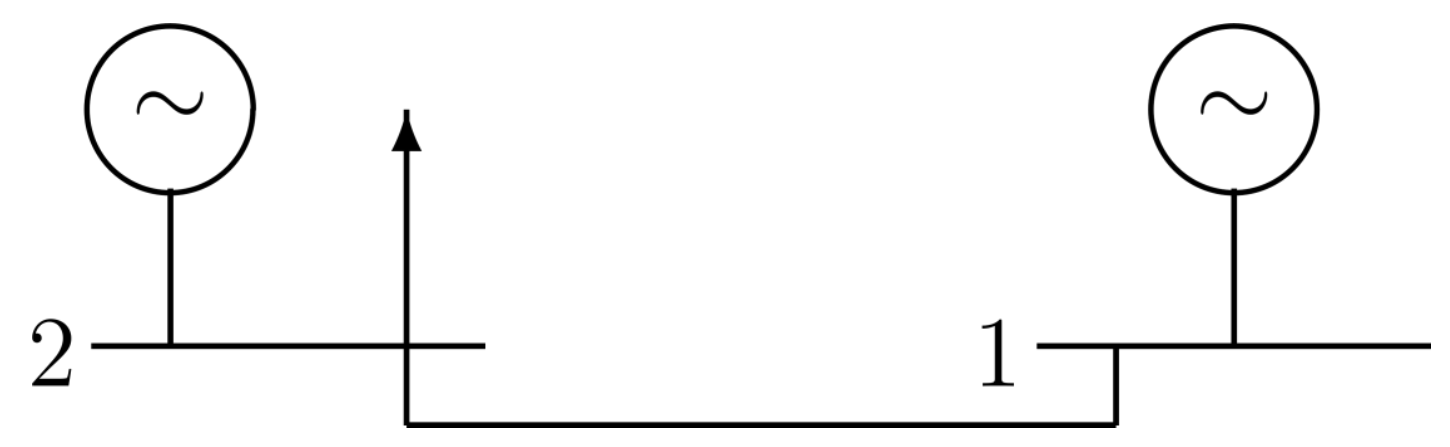

At node 1, a generator of unlimited capacity and per unit production cost of 5 is available, while in node 2 another generator of unlimited capacity and per unit production cost of 10 is available.

The demand is located at node 2 and can take solely two values, either 3 or 5 . 


\section{TEP - Uncertainty}

A robust optimization model for the transmission planner to decide which transmission line to build is: 


\section{TEP - Uncertainty}

$$
\begin{array}{lll}
\min _{x \in\{2,4\}} \max _{d \in\{3,5\}} & \min _{y_{1}, y_{2} \geq 0} & z=1 x+5 y_{1}+10 y_{2} \\
& \text { s.t. } & y_{1}+y_{2}=d \\
& & y_{1} \leq x
\end{array}
$$




\section{TEP - Uncertainty}

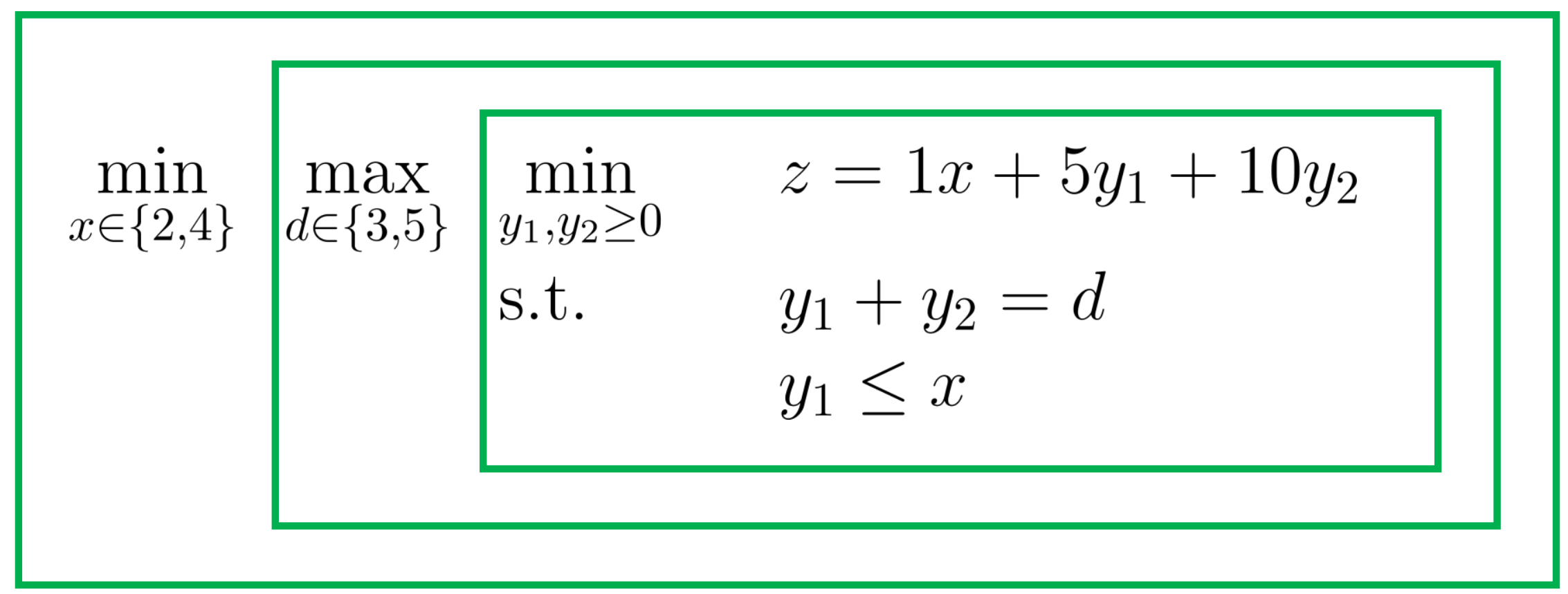




\section{TEP - Uncertainty}

$x$ is the capacity of the transmission line to be built, $d$ the unknown demand, and

$y_{1}$ and $y_{2}$, the production of the units at nodes 1 and 2 , respectively. 


\section{TEP - Uncertainty}

Given the small size and simplicity of this problem, it can be solved by enumeration.

This is done below. 


\section{TEP - Uncertainty}

We first consider that the planner builds the line of capacity 2 , i.e., $x=2$. Then, the problem to be solved is:

$$
\begin{array}{lll}
\max _{d \in\{3,5\}} & \min _{y_{1}, y_{2} \geq 0} & z=2+5 y_{1}+10 y_{2} \\
& \text { s.t. } & y_{1}+y_{2}=d \\
& & y_{1} \leq 2 .
\end{array}
$$




\section{TEP - Uncertainty}

To solve this problem we consider the two alternatives for $d, 3$ and 5 . 


\section{TEP - Uncertainty}

If the demand is $d=3$, the problem to be solved becomes:

$$
\begin{array}{ll}
\min _{y_{1}, y_{2} \geq 0} & z=2+5 y_{1}+10 y_{2} \\
\text { s.t. } & y_{1}+y_{2}=3 \\
& y_{1} \leq 2,
\end{array}
$$

whose solution is $y_{1}=2, y_{2}=1$ and $z=22$. 


\section{TEP - Uncertainty}

On the other hand, if the demand is $d=5$, the problem to be solved becomes:

$$
\begin{array}{ll}
\min _{y_{1}, y_{2} \geq 0} & z=2+5 y_{1}+10 y_{2} \\
\text { s.t. } & y_{1}+y_{2}=5 \\
& y_{1} \leq 2,
\end{array}
$$

whose solution is $y_{1}=2, y_{2}=3$ and $z=42$. 


\section{TEP - Uncertainty}

Considering the above results $(d=3, z=22$ and $d=$ $5, z=42$ ) and the fact that maximum cost is pursued at this level (uncertainty realization), the

solution in case $x=2$ is $d=5$ and $z=42$. 


\section{TEP - Uncertainty}

We then consider that the planner builds the line of capacity 4 , i.e., $x=4$. Then, the problem to be solved is:

$$
\begin{array}{lll}
\max _{d \in\{3,5\}} & \min _{y_{1}, y_{2} \geq 0} & z=4+5 y_{1}+10 y_{2} \\
& \text { s.t. } & y_{1}+y_{2}=d \\
& & y_{1} \leq 4
\end{array}
$$




\section{TEP - Uncertainty}

To solve this problem we consider the two alternatives for $d, 3$ and 5 . 


\section{TEP - Uncertainty}

If the demand is $d=3$, the problem to be solved becomes:

$$
\begin{array}{ll}
\min _{y_{1}, y_{2} \geq 0} & z=4+5 y_{1}+10 y_{2} \\
\text { s.t. } & y_{1}+y_{2}=3 \\
& y_{1} \leq 5,
\end{array}
$$

whose solution is $y_{1}=3, y_{2}=0$ and $z=19$. 


\section{TEP - Uncertainty}

On the other hand, if the demand is $d=5$, the problem to be solved becomes:

$$
\begin{array}{ll}
\min _{y_{1}, y_{2} \geq 0} & z=4+5 y_{1}+10 y_{2} \\
\text { s.t. } & y_{1}+y_{2}=5 \\
& y_{1} \leq 4
\end{array}
$$

whose solution is $y_{1}=4, y_{2}=1$ and $z=34$. 


\section{TEP - Uncertainty}

Considering the above results $(d=3, z=19$ and $d=$ $5, z=34$ ) and the fact that maximum cost is pursued at this level (uncertainty realization),

the solution in case $x=4$ is $d=5$ and $z=34$. 


\section{TEP - Uncertainty}

Finally, considering the two alternatives for investment $x=2(z=42)$ and $x=4(z=34)$, and that the planner seeks minimum cost,

the solution is $x=4, d=5, y_{1}=4, y_{2}=1$ and $z=34$.

The planner builds the larger transmission line to be protected against the high cost of the generator at node 2 . 


\section{TEP - Uncertainty}

A generic adaptive robust optimization problem has the form:

$$
\min _{\mathbf{x} \in \mathcal{X}} \max _{\mathbf{u} \in \mathcal{U}} \min _{\mathbf{y} \in \mathcal{Y}(\mathbf{x}, \mathbf{u})} f(\mathbf{x}, \mathbf{y}, \mathbf{u}) .
$$




\section{TEP - Uncertainty}

An ARO problem comprises three levels. 


\section{TEP - Uncertainty}

$$
\min _{\mathbf{x} \in \mathcal{X}} \max _{\mathbf{u} \in \mathcal{U}} \min _{\mathbf{y} \in \mathcal{Y}(\mathbf{x}, \mathbf{u})} f(\mathbf{x}, \mathbf{y}, \mathbf{u})
$$

The first-level represents a planning strategy prior to the uncertainty realization and seeks to minimize the objective function value.

Decision variables are represented by vector $\mathrm{x} \in \mathcal{X}$. 


\section{TEP - Uncertainty}

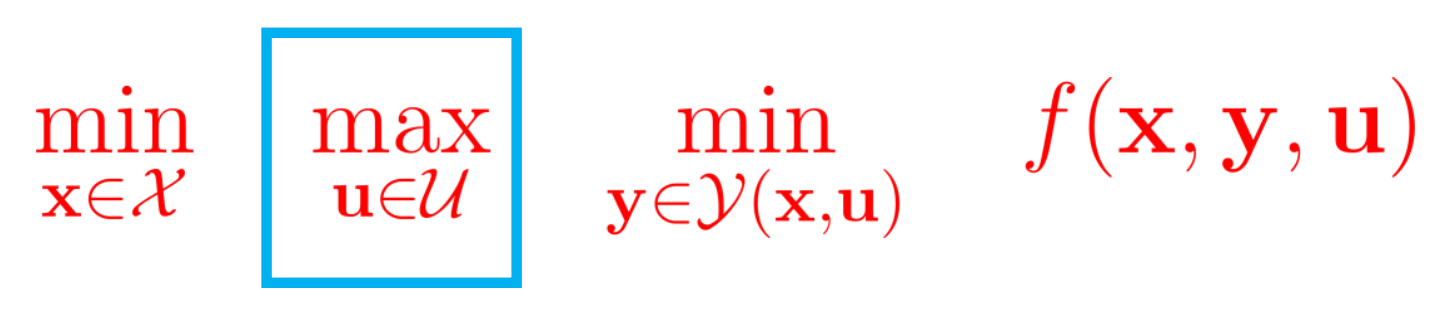

The second-level represents the uncertainty realization in the worst possible manner within an uncertainty set, and thus it seeks to maximize the objective function value.

The variables describing uncertain parameters are represented by vector $\mathbf{u} \in \mathcal{U}$. 


\section{TEP - Uncertainty}

$$
\min _{\mathbf{x} \in \mathcal{X}} \max _{\mathbf{u} \in \mathcal{U}} \min _{\mathbf{y} \in \mathcal{Y}(\mathbf{x}, \mathbf{u})} f(\mathbf{x}, \mathbf{y}, \mathbf{u})
$$

The third-level represents the operation actions to mitigate the pernicious effects of the uncertainty, once it realizes. It therefore seeks to minimize the objective function value.

Decision variables at this level are represented by vector $\mathbf{y} \in \mathcal{Y}(\mathbf{x}, \mathbf{u})$. Note that $\mathcal{Y}$ generally depends on both $\mathbf{x}$ and $\mathbf{u}$. 


\section{TEP - Uncertainty}

The overall objective is to minimize the objective function value under the worst uncertainty realization within the robust set $\mathcal{U}$.

This model provides both preventive and corrective views, i.e., ex-ante protection (planning decisions) and expost correction (operation decisions). 


\section{TEP - Uncertainty}

In expanded form, considering equality and inequality constraints, the problem above becomes:

$$
\begin{array}{llll}
\min _{\mathbf{x} \in \mathcal{X}} & \max _{\mathbf{u} \in \mathcal{U}} & \min _{\mathbf{y} \in \mathcal{Y}} & f(\mathbf{x}, \mathbf{u}, \mathbf{y}) \\
& & \text { s.t. } & \mathbf{h}^{\mathrm{Y}}(\mathbf{x}, \mathbf{u}, \mathbf{y})=\mathbf{0} \\
& & \mathbf{g}^{\mathrm{Y}}(\mathbf{x}, \mathbf{u}, \mathbf{y}) \leq \mathbf{0} \\
\text { s.t. } & \mathbf{h}^{\mathrm{X}}(\mathbf{x})=\mathbf{0} & & \\
& \mathbf{g}^{\mathrm{X}}(\mathbf{x}) \leq \mathbf{0} . & &
\end{array}
$$




\section{Further study}

A. J. Conejo, L. Baringo, S. J. Kazempour, A. S. Siddiqui, "Investment in Electricity Generation and Transmission," Springer, New York. 2016. 
This is it!

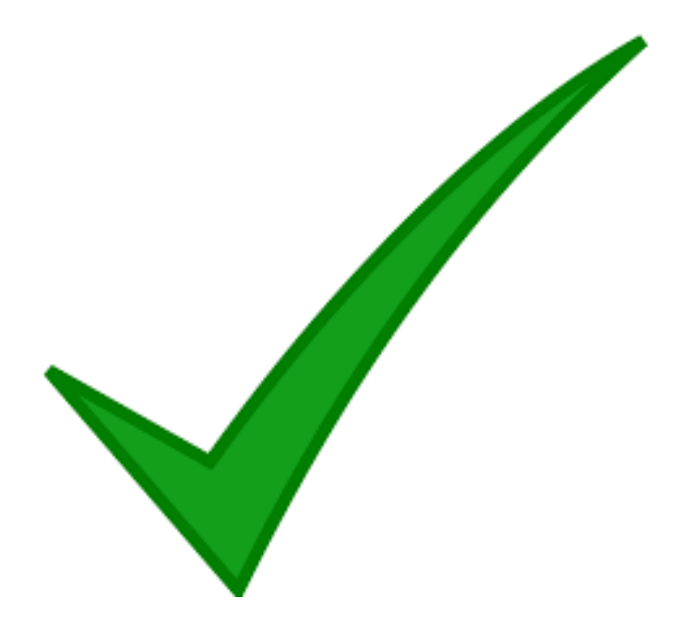

